\title{
Transcriptomic study of high-glucose effects on human skin fibroblast cells
}

\author{
LINGXIA PANG $^{1 *}$, YOUPEI WANG ${ }^{2 *}$, MEIQIN ZHENG ${ }^{2}$, QING WANG $^{1}$, \\ HONG LIN $^{3}$, LIQING ZHANG ${ }^{4}$ and LINGJIAN WU ${ }^{5}$
}

\author{
${ }^{1}$ Function Experiment Teaching Center, Wenzhou Medical University, Wenzhou, Zhejiang 325305; \\ ${ }^{2}$ Clinical Examination Center, The Affiliated Eye Hospital, Wenzhou Medical University, Wenzhou, Zhejiang 325000; \\ ${ }^{3}$ Zhejiang Provincial Key Laboratory of Medical Genetics, Wenzhou Medical University, Wenzhou, Zhejiang 325035; \\ ${ }^{4}$ Operating Room; ${ }^{5}$ Department of Dermatology, The First Affiliated Hospital of Wenzhou Medical University, \\ Wenzhou, Zhejiang 325000, P.R. China
}

Received April 21, 2015; Accepted December 8, 2015

DOI: $10.3892 / \mathrm{mmr} .2016 .4822$

\begin{abstract}
Skin ulcers are a common complication of diabetes mellitus (DM). Fibroblasts are located within the dermis of skin tissue and can be damaged by diabetes. However, the underlying mechanism of how DM affects fibroblasts remains elusive. To understand the effects of DM on fibroblasts, the current study mimicked DM by high-glucose (HG) supplementation in the culture medium of human foreskin primary fibroblast cells, and the analysis of transcriptomic changes was conducted. RNA sequencing-based transcriptome analysis identified that, upon HG stress, 463 genes were upregulated and 351 genes downregulated $(>1.5$-fold changes; $\mathrm{P}<0.05)$. These altered genes were distributed into 20 different pathways. In addition, gene ontology $(\mathrm{GO})$ analysis indicated that $31 \mathrm{GO}$ terms were enriched. Among the pathways identified, nuclear factor $\kappa \mathrm{B}(\mathrm{NF}-\kappa \mathrm{B})$ pathway genes were highly expressed, and the addition of Bay11-7082, a typical NF- $\mathrm{\kappa B}$ signaling inhibitor, blocked the previously observed alterations in plasminogen activator inhibitor 1 (PAI1), an inflammation marker and frizzled class receptor 8 (FZD8), a Wnt signaling gene, expression that resulted from HG stress. Furthermore, an inhibitor of Wnt signaling diminished the role of Bay11-7082 in the regulation of PAI1 expression under HG conditions, suggesting that Wnt signaling may function downstream of the
\end{abstract}

Correspondence to: Professor Meiqin Zheng, Clinical Examination Center, The Affiliated Eye Hospital, Wenzhou Medical University, 268 Xueyuanxi Road, Wenzhou, Zhejiang 325000, P.R. China E-mail:35176404@qq.com

Professor Qing Wang, Function Experiment Teaching Center, Wenzhou Medical University, 268 Xueyuanxi Road, Wenzhou, Zhejiang 325305, P.R. China

E-mail: 33508598@qq.com

${ }^{*}$ Contributed equally

Key words: RNA-Seq, skin, high-glucose, fibroblasts, damage
$\mathrm{NF}-\kappa \mathrm{B}$ pathway to protect fibroblast cells from HG stress. To the best of our knowledge, the current study is the first analysis of transcriptomic responses under HG stress in human fibroblasts. The data provided here may aid the understanding of the molecular mechanisms by which fibroblast cells are damaged in the skin of patients with DM.

\section{Introduction}

Diabetes mellitus (DM) is the most severe metabolic disease in the developed world, affecting a large number of people. A major symptom of DM is hyperglycemia, which leads to severe complications (1). Among patients with DM, 15\% exhibit impaired skin wound healing (2), and high blood sugar is linked to skin ulceration by altering angiogenesis (3), but the underlying mechanism remains unclear.

Skin wound repair requires the coordination of several cell types, including keratinocytes, fibroblasts, endothelial cells, macrophages and platelets. Fibroblast cell proliferation and migration, collagen deposition and remodeling, wound contraction and angiogenesis are important steps during wound repair $(4,5)$. Extracellular matrix (ECM) forms the largest component of the dermal skin layer, therefore, the repair of damaged ECM is a key step for wound healing (6). Fibroblasts constitute one of the important cell layers that participate in the production and remodeling the ECM and fibroblast proliferation and migration are important for the formation of granulation tissue and further skin repair $(7,8)$. The impaired wound healing during DM is attributed to altered protein and lipid metabolism and the associated abnormal formation of granulation tissue (9). Higher glucose levels in the blood result in abnormal attachment of aldose sugars to a protein or lipid, which affects normal glycosylation modifications (9). The aberrantly glycosylated products [advanced glycation end products (AGEs)] then accumulate in cells. AGEs attached to ECM proteins may lead to a reduction in their turnover rate (9). Nitric oxide (NO) is an important mediator of cell proliferation, maturation and differentiation and serves a key role in wound healing (10). Fibroblasts isolated from diabetic ulcers are usually large and widely 
spread during in vitro culture compared with normal fibroblasts in age-matched controls. They often exhibit abnormal endoplasmic reticulum, increased numbers of vesicular bodies and lost microtubular structure. Therefore, DM affects protein turnover, autonomous trafficking and normal protein secretion in diabetic ulcer fibroblasts $(11,12)$. Fibroblasts from diabetic ulcers have defects in cell proliferation, which may result in decreased ECM protein production and further delayed wound healing (13). High glucose-induced fibroblast migration was previously identified to be a result of reduced JNK activity (14). However, few molecular studies have investigating the underlying mechanisms of DM-mediated fibroblast cell damage.

In the present study, RNA sequencing (RNA-Seq) was used to analyze the alterations of large numbers of transcripts following HG stimulation of human fibroblast cells, and the genes and pathways associated with HG stress were identified. Additionally, the inflammatory response pathway and Wnt signaling were further analyzed for their role in the protection of fibroblasts from HG damage. The results of current study may be important for understanding the mechanisms of DM-mediated skin ulceration and may provide a theoretical basis for repair of skin damage in patients with DM in the future.

\section{Materials and methods}

Human foreskin fibroblast cell culture. Human fibroblast cells were isolated and subsequently cultured for analysis of the effects of HG treatment. All the procedures followed for purification and culture of human fibroblasts were described by Xuan et al (14). Human foreskin samples were collected from 3 patients in Department of Dermatology, the First Affiliated Hospital, Wenzhou Medical University (Wenzhou, China). This study was approved by the ethics committee of Wenzhou Medical University (Wenzhou, China) and written informed consent was obtained from all the patients involved. The fat was removed from the tissue and was cut into 3 by $2 \mathrm{~mm}$ strips and were incubated overnight at $4^{\circ} \mathrm{C}$ in $0.05 \%$ Dispase I (Sigma-Aldrich, St. Louis, MO, USA). The epidermis was removed and the dermis was placed in $25-\mathrm{cm}^{2}$ flasks pre-treated with FBS, and placed horizontally for $1 \mathrm{~h}$ and then vertically for $3 \mathrm{~h}$ in a culture chamber with $5 \% \mathrm{CO}_{2}$ at $37^{\circ} \mathrm{C}$. The cells were cultured in Dulbecco's modified Eagle's medium (DMEM; HyClone; GE Healthcare Life Sciences, Logan, UT, USA) containing $5.5 \mathrm{mM}$ glucose with $10 \%$ fetal bovine serum (FBS; HyClone; GE Healthcare Life Sciences) and $1 \%$ penicillin-streptomycin (Gibco; Thermo Fisher Scientific Inc., Watham, MA, USA) the medium was changed every 3 days. When cell confluence reached $70-80 \%$ the cells were digested and passaged with $0.25 \%$ trypsin (Gibco; Thermo Fisher Scientific Inc.) Cells were cultured for 3 days in $5.5 \mathrm{mM}$ glucose medium and transferred to the media containing either $5.5 \mathrm{mM}$ glucose (LG) or $30 \mathrm{mM}$ glucose (HG). Cells at passage 3-6 were used for the LG and HG treatment. Cells were harvested after $1 \mathrm{~h}$ of LG and HG treatment.

Cell proliferation assay. Cell proliferation was assayed using a Cell Counting Kit-8 (CCK-8) kit (Dojindo Molecular Technologies, Inc., Kumamoto, Japan). The fibroblast cell culture and measurement of cell densities under different
Table I. Reverse transcription-quantitative polymerase chain reaction primer sequences.

\begin{tabular}{ll}
\hline Primer & \multicolumn{1}{c}{ Sequence } \\
\hline Caspase 3 & F: TGATGATGACATGGCGTGTC \\
& R: GTTGCCACCTTTCGGTTAAC \\
FAI1 & R: GAGACTGAAGTCGACCTCAG \\
& F: CTGTCCATGATGATCTCCTC \\
GAPDH & R: CTGTAGCCAAATTCGTTGTC \\
& F: CGTCCCATCTACTTGCTGCT \\
CCL13 & R: TCAAGTCTTCAGGGTGTGAGC \\
& F: GGTGCAGTTTTGCCAAGGAG \\
IL8 & R: TTCCTTGGGGTCCAGACAGA \\
& F: CTGGTGGAGATCCAGTGCTC \\
FZD8 & R: TTGTAGTCCATGCACAGCGT \\
& F: TCGCAAGTACCCCAACAGAC \\
EGR2 & R: CTCATCACTCCGGGCAAACT
\end{tabular}

PAI1, plasminogen activator inhibitor 1; GAPDH, glyceraldehyde-3-phosphate dehydrogenase; CCL13, chemokine (C-C motif) ligand 13; IL8, interleukin 8; FZD8, frizzled class receptor 8; EGR2, early growth response 2 .

treatments were followed as previously described (14). In summary, $50 \mathrm{ml}$ of the cell resuspension solution (1x10 ${ }^{3}$ cells/well) were transferred into 96 -well plates following digestion with trypsin, and five parallel wells were used for each treatment. Subsequent to attachment to the culture plate, the cells were subjected to the different glucose treatments for $72 \mathrm{~h}$ in a $5 \% \mathrm{CO}_{2}$ incubator at $37^{\circ} \mathrm{C}$. Then, $5 \mathrm{ml}$ of CCK-8 was added to each well, and the cells were cultured for another $3 \mathrm{~h}$. Cell density was determined by quantifying the absorbance at $450 \mathrm{~nm}$ using a Varioskan Flash Multimode Reader (Thermo Fisher Scientific, Inc.) using the following formula: Cell dens ity $=\left(\mathrm{A}_{\text {cell+CCK8+medium }}-\mathrm{A}_{\mathrm{CCK} 8+\text { medium }} / \mathrm{A}_{\text {cell+CCK8 }}-\mathrm{A}_{\mathrm{CCK} 8+\text { medium }}\right) \times 100$.

RNA deep sequencing. Total RNA was extracted from human foreskin fibroblasts for RNA-Seq experiments following treatment with a low $(5.5 \mathrm{mM})$ or high $(30 \mathrm{mM})$ concentration of glucose. RNA-Seq experiments and data analysis were performed by the NovelBio Bio-Pharm Technology Co., Ltd. (Shanghai, China). The RNA-Seq data is available upon request.

Analysis of the pathway and gene ontology (GO) category. Differentially expressed genes were identified by analyzing for association with biological process gene ontology (GO) terms (15). Fisher's exact test was used to classify the GO category, and the false discovery rate (FDR) was calculated to correct the P-value (16). Enrichment of GO members among differentially expressed gene sets was identified using the one-tailed Fisher's exact test for 2x2 contingency tables (17), which measures the significance of the function that as the enrichment increases, the corresponding function is more specific, which aids the identification of GOs with a more 
A

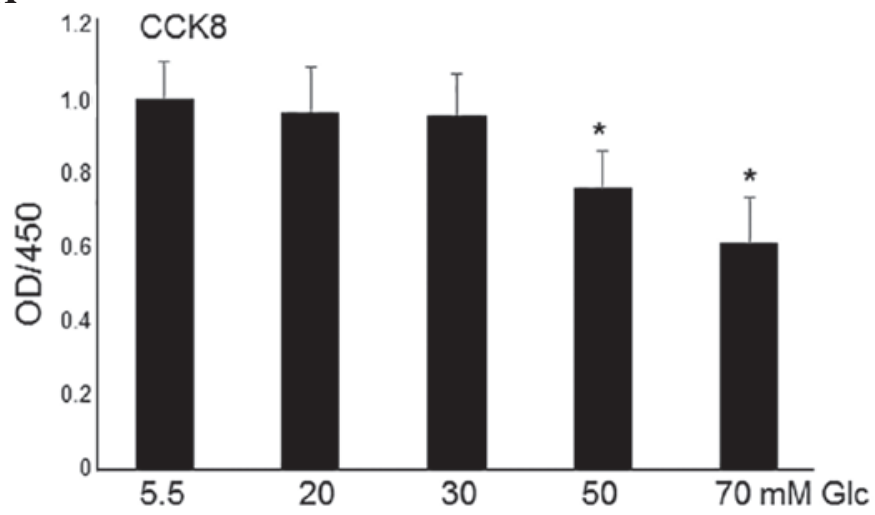

B

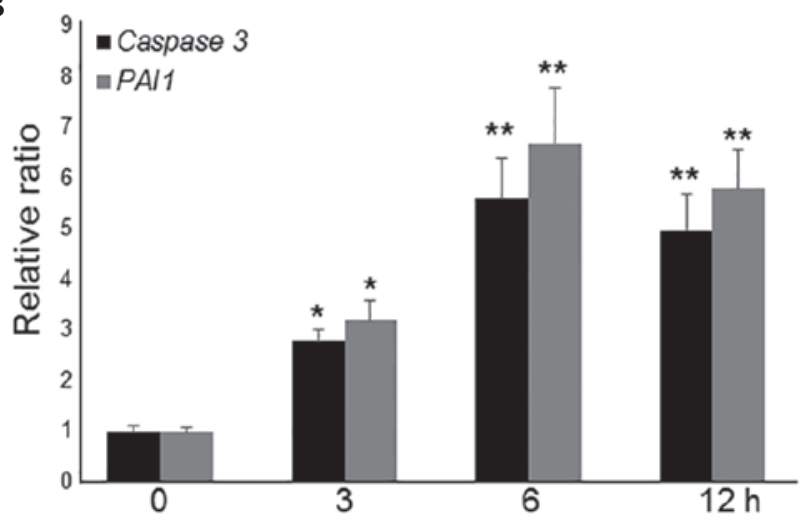

Figure 1. High-Glc effects on fibroblast cells. (A) Cell proliferation under the high-Glc conditions with $10 \%$ fetal bovine serum was measured by cell counting kit- 8 assay after a 72 -h incubation ( $\mathrm{P}<0.01 \mathrm{vs}$. the $5.5 \mathrm{mM}$ Glc group; t-test). (B) Reverse transcription-quantitative polymerase chain reaction was performed to monitor the expression levels of caspase 3 and PAI1 following high-dose Glc $(30 \mathrm{mM})$ treatment. GAPDH was used as an internal control. Data represent the mean \pm standard error of 3 replicates. ${ }^{*} \mathrm{P}<0.05$ and ${ }^{* *} \mathrm{P}<0.01$ vs. the 0 h group; t-test. Glc, glucose; OD, optical density; PAI1, plasminogen activator inhibitor 1 .

concrete function description in the experiment. Pathway analysis was used to determine the significant pathways of the differential genes according to Kyoto Encyclopedia of Genes and Genomes (KEGG) (18), BioCarta (http://cgap.nci.nih.gov/Pathways/BioCarta_Pathways and Reactome(19).Fisher'sexacttestwasfollowedbyBenjamini-Hochberg multiple testing correction to select the significant pathway and the threshold of significance was defined by P-value and FDR (20).

Total RNA extraction, cDNA synthesis and reverse transcription-quantitative polymerase chain reaction $(R T-q P C R)$. Total RNA was extracted from stimulated or unstimulated fibroblasts treated with high-concentration glucose (30 mM), Bay11-7082 (0.5 $\mu \mathrm{M}$; Sigma-Aldrich) or inhibitor of Wnt response (IWR) $(0.5 \mu \mathrm{M}$; Sigma-Aldrich). The cell monolayer was rinsed with ice-cold phosphate-buffered saline once. Each sample was treated with RQ1-DNAse (Promega Corporation, Madison, WI, USA). The cells were then lysed directly in a culture dish by adding $1 \mathrm{ml}$ TRIzol (Thermo Fisher Scientific, Inc.) per each $3.5 \mathrm{~cm}$ diameter dish, scraped with a cell scraper and then $0.2 \mathrm{ml}$ chloroform was added per $1 \mathrm{ml}$ TRIzol. RNA $(2 \mu \mathrm{g})$ was reverse transcribed at $42^{\circ} \mathrm{C}$ for $60 \mathrm{~min}$, $70^{\circ} \mathrm{C}$ for $5 \mathrm{~min}$ and following stop the reaction at $8^{\circ} \mathrm{C}$ using a GoScript Reverse Transcription System (Promega Corporation) following the manufacturer's protocol. A SYBR Green Master Mix (Bio-Rad Laboratories, Inc., Hercules, CA, USA) was used to perform the qPCR on an Illumina Eco 3.0 (Illumina, Inc., San Diego, CA, USA). A typical reaction consisted of an initial denaturation at $95^{\circ} \mathrm{C}$ for $3 \mathrm{~min}$, followed by 40 cycles of denaturation for $30 \mathrm{sec}$ at $95^{\circ} \mathrm{C}$, annealing for $30 \mathrm{sec}$ at $58^{\circ} \mathrm{C}$, and extension at $72^{\circ} \mathrm{C}$ for $30 \mathrm{sec}$, followed by a final extension at $72^{\circ} \mathrm{C}$ for $5 \mathrm{~min}$. The transcription levels were normalized against those of GAPDH using the $2^{-\Delta \Delta \mathrm{Cq}}$ method (21). The gene-specific primer sequences used for RT-qPCR are described in Table I. Each experiment was repeated at least 3 times. A unreversed transcribed RNA was used as a PCR template control.

Western blot analysis. For extraction of total protein, the cells were lysed in an ice-cold lysis solution containing $7 \mathrm{M}$ urea,
2 Mthiourea, 2\% CHAPS detergent, $40 \mathrm{mM}$ Trizma base, $40 \mathrm{mM}$ dithiothreitol, $1 \%$ protease inhibitor, the lysates were centrifuged for $15 \mathrm{~min}$ at $15,000 \mathrm{x}$ g. All reagents were sourced from Sigma-Aldrich. The supernatant from each tube was moved to a new tube. The total proteins were separated on a $10 \%$ sodium dodecyl sulfate polyacrylamide gel electrophoresis gel (Sigma-Aldrich) at $100 \mathrm{~V}$ for $2 \mathrm{~h}$ following extraction. Then transferred onto Immobilon-P Transfer Membranes (Merck Millipore, Tokyo, Japan). The membranes were incubated in Tris-buffered saline containing 5\% skimmed milk and $0.05 \%$ Tween-20 (EMD Milipore, Billerica, MA, USA) for 1-2 $\mathrm{h}$ and reacted with the corresponding primary antibodies at $4^{\circ} \mathrm{C}$ overnight. The following primary antibodies were purchased from Abcam, all at dilution of 1:2,000 (Cambridge, MA, USA): p-IKB $\alpha$ (mouse monoclonal; cat no. 39A1431, reactivity - mouse, rat, cow, human), IKB $\alpha$ (rabbit polyclonal; cat no. ab7217; reactivity - mouse, rat, human) and GAPDH (mouse monoclonal; cat no. mAbcam 9484; reactivity - mouse, rat, rabbit, chicken, cow, dog, human, pig). The membranes were incubated for $1 \mathrm{~h}$ with an anti-mouse or polyclonal anti-rabbit horseradish peroxidase-linked secondary antibody (cat. no. 7074; 1:2,000; Cell Signaling Technology, Inc., Danvers, MA, USA).

Statistical analysis. Statistical calculations were performed with Prism 5 software package (GraphPad Software, Inc., La Jolla, CA, USA). Significant differences are expressed as the mean \pm standard error. The comparison between two groups was analyzed by t-test. $\mathrm{P}<0.05$ was considered to indicate a statistically significant difference.

\section{Results}

$H G$ induces apoptosis and inflammatory responses in fibroblasts. To simulate diabetes, HG was utilized to study its effects on the fibroblasts (22). To analyze the effects of different concentrations of $\mathrm{HG}$ on fibroblasts, cell proliferation was monitored in different concentrations of glucose-containing media with $10 \%$ fetal bovine serum. HG treatment up to $30 \mathrm{mM}$ did not markedly alter cell proliferation, whilst culture in 50 and $70 \mathrm{mM}$ glucose significantly inhibited cell 
Table II. GO classification.

$\mathrm{Up} /$

GO ID

GO term

Enrichment

$(-\log 2 \mathrm{P})$

downregulated

0071371

0034421

0071320

0001508

0050890

0034088

0006642

0035356

0071356

0042471

0042158

0045444

0048752

0030728

0006955

0006351

0042472

0010875

0002237

0006334

0035115

0007275

0032688

0060675

2001181

0048706

0009952

0060070

0010042

0009954

0046628
Cellular response to gonadotropin stimulus

Post-translational protein acetylation

Cellular response to cAMP

Regulation of action potential

Cognition

Maintenance of mitotic sister chromatid cohesion

Triglyceride mobilization

Cellular triglyceride homeostasis

Cellular response to tumor necrosis factor

Ear morphogenesis

Lipoprotein biosynthetic process

Fat cell differentiation

Semicircular canal morphogenesis

Ovulation

Immune response

Transcription, DNA-templated

Inner ear morphogenesis

Positive regulation of cholesterol efflux

Response to molecule of bacterial origin

Nucleosome assembly

Embryonic forelimb morphogenesis

Multicellular organism development

Negative regulation of interferon-beta production

Ureteric bud morphogenesis

Positive regulation of interleukin-10 secretion

Embryonic skeletal system development

Anterior/posterior pattern specification

Canonical Wnt signaling pathway

Response to manganese ion

Proximal/distal pattern formation

Positive regulation of insulin receptor signaling pathway

\begin{tabular}{llc}
19.12899 & 10.23295 & $\mathrm{Up}$ \\
63.76331 & 9.872556 & $\mathrm{Up}$ \\
9.564497 & 9.733907 & $\mathrm{Up}$ \\
15.94083 & 9.562348 & $\mathrm{Up}$ \\
15.10184 & 9.362226 & $\mathrm{Up}$ \\
47.82249 & 9.297728 & $\mathrm{Up}$ \\
38.25799 & 8.822425 & $\mathrm{Up}$ \\
38.25799 & 8.822425 & $\mathrm{Up}$ \\
7.501566 & 8.538068 & $\mathrm{Up}$ \\
31.88166 & 8.417503 & $\mathrm{Up}$ \\
31.88166 & 8.417503 & $\mathrm{Up}$ \\
6.596205 & 7.912633 & $\mathrm{Up}$ \\
23.91124 & 7.753206 & $\mathrm{Up}$ \\
23.91124 & 7.753206 & $\mathrm{Up}$ \\
2.610657 & 7.576953 & $\mathrm{Up}$ \\
1.628693 & 7.526714 & $\mathrm{Up}$ \\
5.710147 & 7.220235 & $\mathrm{Up}$ \\
17.38999 & 6.989887 & $\mathrm{Up}$ \\
17.38999 & 6.989887 & $\mathrm{Up}$ \\
7.598543 & 21.94307 & Down \\
12.5653 & 13.38364 & Down \\
2.149419 & 11.51722 & Down \\
53.61194 & 9.387513 & Down \\
53.61194 & 9.387513 & Down \\
53.61194 & 9.387513 & Down \\
8.247991 & 8.969668 & Down \\
4.684538 & 8.623385 & Down \\
5.154994 & 8.014638 & Down \\
26.80597 & 7.938039 & Down \\
10.05224 & 7.809469 & Down \\
22.97655 & 7.587427 & Down \\
\hline & & \\
& & \\
& & \\
& & \\
& &
\end{tabular}

GO, gene ontology.

proliferation compared with culture in low-glucose (LG; $5.5 \mathrm{mM}$; $\mathrm{P}<0.01$; Fig. 1A). However, this concentration of glucose is much higher than the levels recorded in patients' blood; therefore, 20 and $30 \mathrm{mM}$ were used to further analyze gene expressions. Compared to $30 \mathrm{mM}, 20 \mathrm{mM}$ of glucose did not significantly affect the expression levels of caspase 3 and PAIl (data not shown). Therefore, $30 \mathrm{Mm}$ of glucose was selected for transcriptome analysis. As presented in Fig. 1A, $30 \mathrm{mM}$ glucose treatment did not affect cell proliferation activity, thus, the expression levels of two apoptosis and inflammation marker genes, caspase 3 and plasminogen activator inhibitor 1 (PAI1), were further monitored at this concentration. RT-qPCR results indicated that the application of $30 \mathrm{mM}$ glucose led to an increase in the expression levels of caspase 3 and PAI1 following 3 -h treatment, which reached a peak at $6 \mathrm{~h}$ (Fig. 1B). These data indicate that HG culture damages fibroblast cells.
Identification of HG-regulating transcriptome in fibroblasts. To identify HG-regulated genes and pathways, RNA-Seq experiments were performed using human fibroblast cells cultured in LG $(5.5 \mathrm{mM})$ and $\mathrm{HG}(30 \mathrm{mM})$. Since Caspase 3 and PAIl expression levels were highest at $6 \mathrm{~h}$ subsequent to HG stress, the fibroblast cells stimulated for $6 \mathrm{~h}$ with $\mathrm{LG}$ and HG were collected for RNA-Seq analysis. The RNA-Seq results demonstrated that 814 genes were differentially expressed $(>1.5$-fold change; $\mathrm{P}<0.05)$ in the HG-treated fibroblasts compared with LG-treated cells. Among them, 351 genes were downregulated, and 463 genes were upregulated (Fig. 2A), determined from statistical outcomes by analysis for association with biological process GO terms. To verify the RNA-Seq data, HG-mediated expression levels of the following four genes were assessed by RT-qPCR: Interleukin 8 (IL8), chemokine (C-C motif) ligand 13 (CCL13), frizzled class receptor 8 (FZD8) and early growth receptor 2 
A

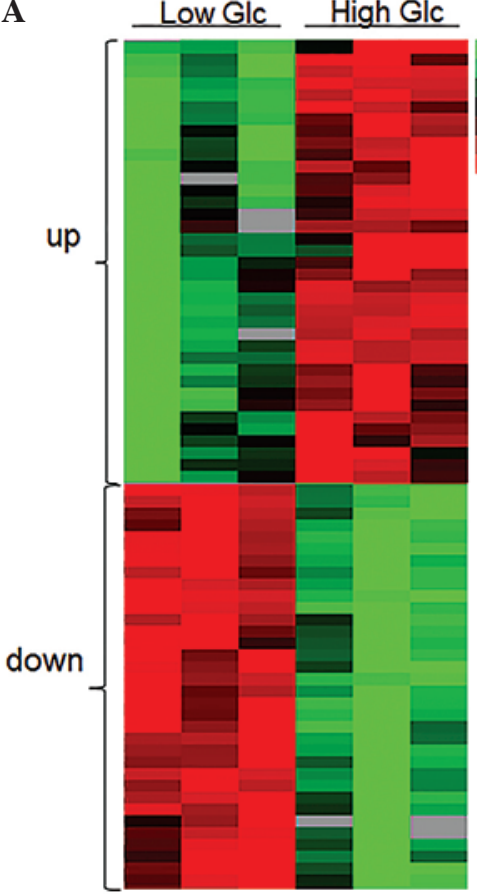

B
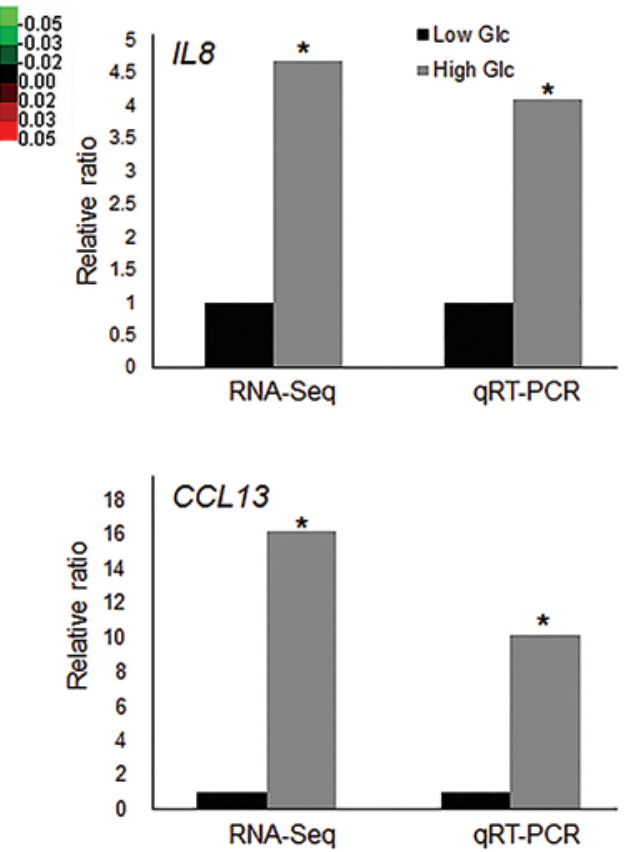
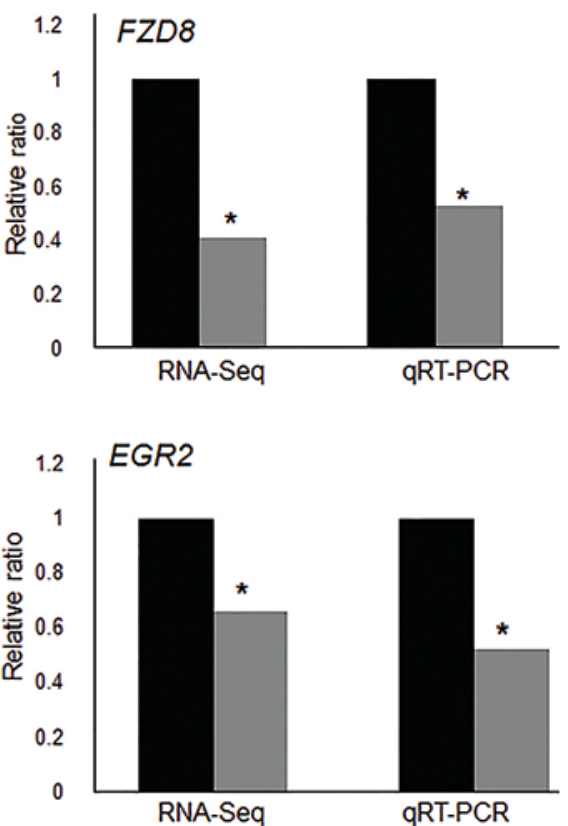

Figure 2. High Glc regulated transcriptome profile. (A) Heat map represents the differentially expressed genes following high Glc $(30 \mathrm{mM})$ treatment for $6 \mathrm{~h}$ in human fibroblast cells. Low Glc treatment was $5.5 \mathrm{mM}$. Gene expression is presented as a pseudocolor scale with red denoting higher gene expression levels and green denoting lower levels. Significant differences between low and high glucose treated groups were compared $(\mathrm{P}<0.05)$. (B) RT-qPCR was performed to verify the expression levels of IL8, CCL13, FZD8 and EGR2 and the data was compared with RNA-Seq results. Significant differences of IL8, FZD8, CCL13 and EGR2 expression levels between low and high glucose treated groups in both RNA-Seq and qRT-PCR analyses ("P $<0.05)$ GAPDH was used as an internal control. Glc, glucose; RT-qPCR, reverse transcription-quantitative polymerase chain reaction; IL8, interleukin 8; FZD8, frizzled class receptor 8; CCL13, chemokine (C-C motif) ligand 13; EGR2, early growth response 2; RNA-Seq, RNA sequencing.

A

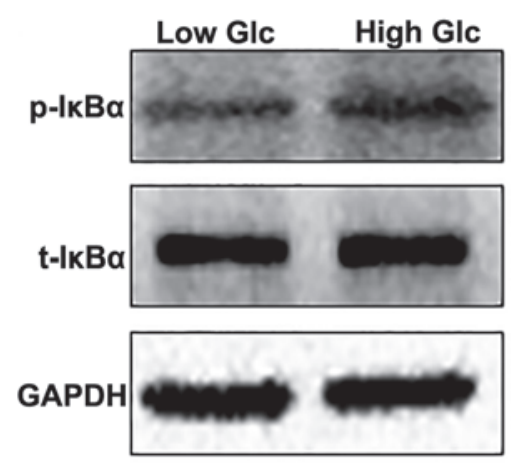

B

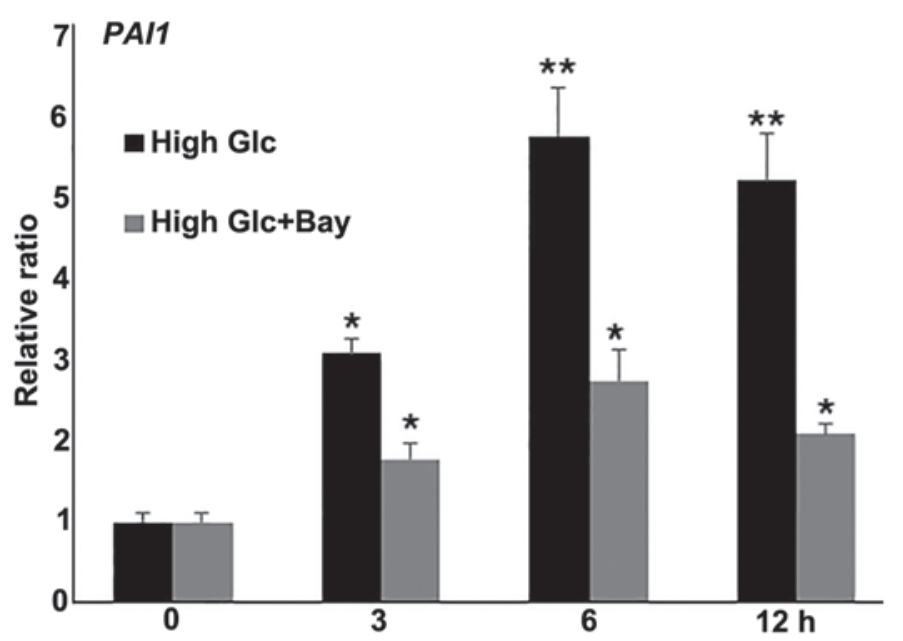

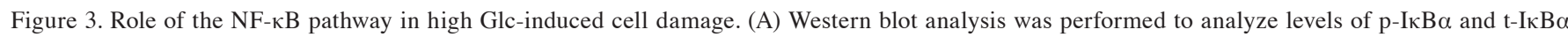
following high Glc stress for $1 \mathrm{~h}$. (B) Reverse transcription-quantitative polymerase chain reaction was performed to monitor the expression levels of PAI1 following high Glc or high Glc + Bay $(0.5 \mu \mathrm{M})$ treatment. GAPDH was used as an internal control. Data represent the mean \pm standard error of 3 replicates.

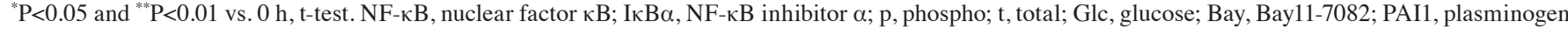
activator inhibitor 1 .

(EGR2). The inflammatory response genes (IL8 and CCL13) were upregulated, while the Wnt signaling gene (FZD8) and putative SUMO E3 ligase (EGR2) were repressed by HG stimulation, and the RT-qPCR results were similar to RNA-Seq data (Fig. 2B). GO analysis indicated that $31 \mathrm{GO}$ terms were enriched $(\mathrm{P}<0.01$; Table II). These genes were associated with multiple biological processes, including cellular triglyceride homeostasis, positive regulation of cholesterol efflux, the canonical Wnt signaling pathway and transcription (Table II). Further pathway analysis was performed with 814 genes that were altered by HG stress. The results demonstrated that these genes were divided into 20 different pathways, including NF- $\kappa B$, tumor necrosis factor (TNF), Wnt, ECM/receptor interaction and hedgehog signaling pathways. Among them, 
Table III. Pathway classification.

\begin{tabular}{|c|c|c|c|c|}
\hline Pathway ID & Pathway term & Enrichment & $(-\log 2 \mathrm{P})$ & downregulatec \\
\hline 05034 & Alcoholism & 8.39875 & 32.3993 & Up \\
\hline 05322 & Systemic lupus erythematosus & 9.129076 & 29.151 & Up \\
\hline 05217 & Basal cell carcinoma & 7.635227 & 10.23381 & Up \\
\hline 04512 & ECM-receptor interaction & 4.772017 & 7.471521 & Up \\
\hline 04916 & Melanogenesis & 4.157797 & 6.698221 & Up \\
\hline 04390 & Hippo signaling pathway & 3.27224 & 6.165099 & Up \\
\hline 05203 & Viral carcinogenesis & 2.840157 & 5.914846 & Up \\
\hline 05202 & Transcriptional misregulation in cancer & 2.799583 & 5.229064 & Up \\
\hline 04910 & Insulin signaling pathway & 2.957306 & 4.892077 & Up \\
\hline 04340 & Hedgehog signaling pathway & 4.49933 & 4.869559 & Up \\
\hline 04977 & Vitamin digestion and absorption & 6.998958 & 4.700239 & Up \\
\hline 04064 & $\mathrm{NF}-\kappa \mathrm{B}$ signaling pathway & 6.15293 & 8.938564 & Up \\
\hline 04668 & TNF signaling pathway & 5.090152 & 7.82519 & Up \\
\hline 04060 & Cytokine-cytokine receptor interaction & 3.803208 & 9.593708 & Down \\
\hline 04310 & Wnt signaling pathway & 2.74469 & 4.521127 & Down \\
\hline 05202 & Transcriptional misregulation in cancer & 3.732778 & 6.955703 & Down \\
\hline 05166 & HTLV-I infection & 2.94693 & 6.11439 & Down \\
\hline 04621 & NOD-like receptor signaling pathway & 5.418548 & 5.528479 & Down \\
\hline 05132 & Salmonella infection & 3.952353 & 4.424635 & Down \\
\hline 05161 & Hepatitis B & 3.068037 & 4.341831 & Down \\
\hline
\end{tabular}

ECM, extracellular matrix; NF- $\mathrm{BB}$, nuclear factor $\kappa \mathrm{B}$; TNF, tumor necrosis factor; HTLV-I, human T-lymphotropic virus 1; NOD, nucleotide-binding oligomerization domain.

A

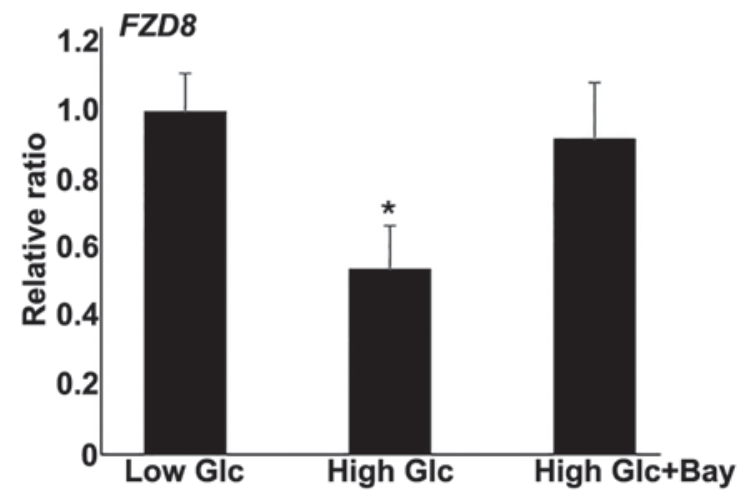

B

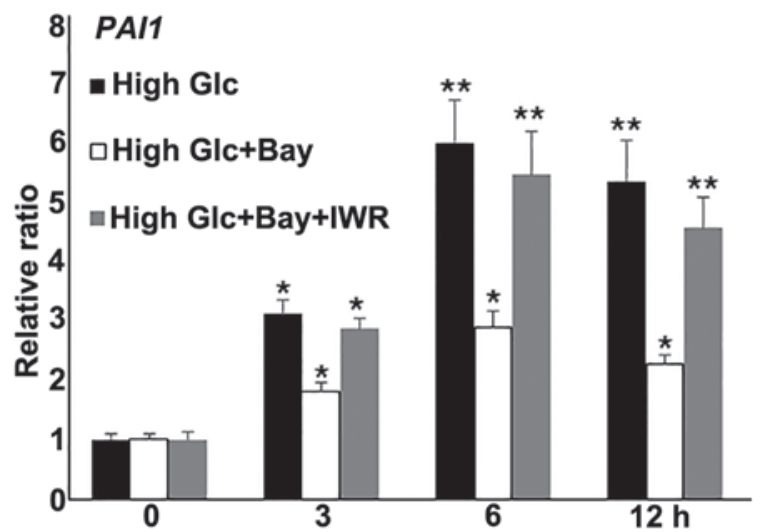

Figure 4. Effect of Wnt signaling pathway on high Glc-mediated cell damage. (A) RT-qPCR was performed to analyze expression level of Wnt signaling gene FZD8 after high Glc and high Glc + Bay treatment. "P<0.05 vs. low Glc; t-test. (B) RT-qPCR analysis for monitoring PAI1 expressions after Bay or Bay + IWR (a typical Wnt signaling inhibitor treatment) at the indicated time points. " $\mathrm{P}<0.05$ and ${ }^{* * *} \mathrm{P}<0.01 \mathrm{vs.} 0 \mathrm{~h}$; t-test. Glc, glucose; RT-qPCR, reverse transcription-quantitative polymerase chain reaction; FZD8, frizzled class receptor 8; Bay, Bay11-7082; PAI1, plasminogen activator inhibitor 1; IWR, inhibitor of Wnt response.

certain inflammatory response pathways involving NF- $\mathrm{B}$ and TNF were upregulated by HG stress, while Wnt signaling genes were downregulated (Table III). Together, these data indicate that $\mathrm{HG}$ regulates a large number of genes involved in various biological processes.

Regulatoryrole of the inflammatory response in $H G$-mediated fibroblast cell damage. The NF- $\mathrm{kB}$ pathway was identified to be involved in HG-regulated biological processes, the effect of the inflammatory response in HG-mediated fibroblast cell damage was further examined. To further evaluate the effects of $\mathrm{HG}$ on $\mathrm{NF}-\kappa \mathrm{B}$ signaling, the activity of I $\kappa \mathrm{B} \alpha$, the most characterized and studied NF- $\kappa \mathrm{B}$ regulator, was

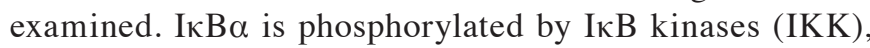
resulting in the translocation of NF- $\mathrm{KB}$ to the nucleus and transcription of target genes (23). Western blot analysis 


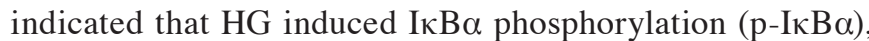

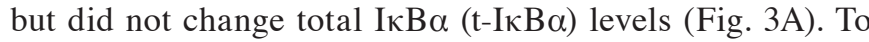
further analyze the effect of the inflammatory response on HG-mediated gene expression, a combination of HG stress and Bay11-7082 $(0.5 \mu \mathrm{M})$, a representative NF- $\kappa \mathrm{B}$ pathway inhibitor, was used to treat fibroblasts. RNA was extracted and RT-qPCR was performed to monitor PAIl gene expression. The results indicated that $\mathrm{HG}$ induced an increase in PAI1 mRNA levels at $3(\mathrm{P}<0.05), 6$ and $12 \mathrm{~h}(\mathrm{P}<0.01)$ compared with the levels observed at $0 \mathrm{~h}$, and this induction was blocked by inhibiting NF-אB with Bay11-7082 (Fig. 3B). Taken together, these results suggest that the inflammatory response is inversely correlated with HG-regulated gene expression.

Wnt signaling is downstream of the $N F-\kappa B$ pathway. Wnt signaling is known to regulate diverse aspects of numerous biological processes (24). The RNA-Seq data from the present study demonstrated repressed expression of a number of Wnt signaling genes following HG stimulation in fibroblasts. NF- $\mathrm{KB}$ pathway inhibition partially rescued HG-mediated fibroblast damage. Therefore, the relationship between NF- $\mathrm{\kappa B}$ and Wnt signaling were investigated further. In the fibroblast cells stimulated with Bay11-7082 $(0.5 \mu \mathrm{M})$ and HG, expression levels of the Wnt signaling gene, FZD8, were similar to the levels in the LG cells ( $\mathrm{P}>0.05$; Fig. 4A). To further evaluate the role of Wnt signaling in HG-mediated gene expression, IWR, a typical Wnt signaling inhibitor, was also used to treated fibroblasts alongside Bay11-7082 and HG culture, then PAI1 gene expression was measured. Notably, IWR application reduced the effect of Bay11-7082 on HG-induced PAI1 expression levels (Fig. 4B). Together, these data demonstrate that NF- $\mathrm{\kappa B}$ inhibition blocked the gene expression changes induced by HG. Additionally, Wnt signaling inhibition reversed Bay11-7082-mediated PAI1 repression under HG conditions.

\section{Discussion}

Fibroblasts are important for synthesizing ECM and collagen, the structural framework (stroma) for skin tissues, and serve a key role in wound healing (25). Skin wound healing requires the involvement of several cell types, including keratinocytes, fibroblasts, endothelial cells, macrophages and platelets (26). Therefore, understanding the underlying mechanisms by which fibroblast cells protect themselves from DM is important for the treatment of skin ulcers (4). One of the strategies that all living organisms utilize to adapt to environmental changes is the rapid reprogramming of transcriptional regulations via cell signaling mechanisms (27-29). Therefore, analysis of transcriptomic changes under certain stress is a method to clarify the regulation of these mechanisms. In the present study, RNA-Seq was utilized to analyze transcriptomes, providing an efficient experimental basis to extract information regarding gene expression, somatic mutations and novel gene fusions (30) using HG-cultured human primary fibroblast cells. The results demonstrated a large population of differentially expressed genes following HG stimulation. Among them, 351 genes were downregulated and 463 were upregulated. Further, analyses of the associated pathways using GO and KEGG databases revealed various biological processes and pathways (Tables II and III).

ECM synthesis is important for skin wound repair. Various genes involved in $\mathrm{ECM} /$ receptor interactions were identified as undergoing changes in expression levels following HG stress (data available upon request). Other pathways identified to be altered by HG include NF-кB, TNF, Wnt, Hedgehog and Hippo signaling. The Wnt signaling pathway and fibroblast growth factor (FGF) regulate T-box family transcription factors, control cell fate within the zebrafish tailbud and are involved in axis elongation (31); FGF positively regulates Hedgehog signaling during embryonic tracheal cell migration (32); Hippo signaling and EGFR pathways control growth and activate tumorigenesis when dysregulated. Epidermal growth factor receptor (EGFR) activates Yorkie, a key Hippo pathway transcription factor that has been indicated to influence cell proliferation in Drosophila (33); and bFGF previously inhibited TNF-mediated activation of NF- $\mathrm{BB}$ by blocking phosphorylation and degradation of $\mathrm{I} \kappa \mathrm{B} \alpha$, leading to the repression of leukocyte adhesion in tumor vessels (34). HG has been demonstrated to affect FGF and downstream JNK activity, resulting in delay to human fibroblast cell migration (14). Therefore, those pathways that are regulated by HG may be partially connected to FGF signaling, which is known to accelerate DM-induced skin wound repair. Further biochemical and molecular studies are required to specify how these pathways are connected.

In the current study, inhibition of the NF- $\mathrm{kB}$ pathway through treatment with Bay11-7082 repressed the HG-induced PAI1 levels, suggesting that HG stimulation may activate inflammatory response pathways and cause damage to cells. Notably, Bay11-7082 application reversed the repression of FZD8 expression levels resulting from HG stress.

In addition, treatment with the Wnt signaling inhibitor, IWR, together with Bay11-7082 diminished the effects of Bay11-7082 on PAI1 repression under HG conditions, indicating that Wnt signaling functions downstream of the NF- $\mathrm{kB}$ pathway to regulate HG-mediated gene expression. HG stress negatively and positively regulated the NF- $\mathrm{kB}$ and Wnt signaling pathways, respectively, and this suggests that Wnt activation is important for the protection of fibroblasts from DM. The present study demonstrated HG-regulated gene expression in fibroblasts, and a link between the NF- $\mathrm{KB}$ pathway and Wnt signaling. In the future, these findings may be notable for the treatment of DM-induced skin ulcers.

\section{Acknowledgements}

The present study was made possible by an initiative grant from Wenzhou Medical University (Wenzhou, China).

\section{References}

1. Brownlee M: Biochemistry and molecular cell biology of diabetic complications. Nature 414: 813-820, 2001.

2. Yach D, Stuckler D and Brownell KD: Epidemiologic and economic consequences of the global epidemics of obesity and diabetes. Nat Med 12: 62-66, 2006.

3. Braiman-Wiksman L, Solomonik I, Spira R and Tennenbaum T: Novel insights into wound healing sequence of events. Toxicol Pathol 35: 767-779, 2007.

4. Martin P: Wound healing - aiming for perfect skin regeneration. Science 276: 75-81, 1997 . 
5. Gurtner GC, Werner S, Barrandon Y and Longaker MT: Wound repair and regeneration. Nature 453: 314-321, 2008.

6. Brem $\mathrm{H}$ and Tomic-Canic M: Cellular and molecular basis of wound healing in diabetes. J Clin Invest 117: 1219-22, 2007.

7. Wagner W and Wehrmann M: Differential cytokine activity and morphology during wound healing in the neonatal and adult rat skin. J Cell Mol Med 11: 1342-1351, 2007.

8. Kanazawa S, Fujiwara T, Matsuzaki S, Shingaki K, Taniguchi M, Miyata S, Tohyama M, Sakai Y, Yano K, Hosokawa K and Kubo T: bFGF regulates PI3-kinase-Rac1-JNK pathway and promotes fibroblast migration in wound healing. PLoS One 5: e12228, 2010

9. Goldin A, Beckman JA, Schmidt AM and Creager MA: Advanced glycation end products: Sparking the development of diabetic vascular injury. Circulation 114: 597-605, 2006.

10. Obayashi K, Akamatsu H, Okano Y, Matsunaga K and Masaki H: Exogenous nitric oxide enhances the synthesis of type I collagen and heat shock protein 47 by normal human dermal fibroblasts. J Dermatol Sci 41: 121-126, 2006.

11. Loots MA, Lamme EN, Mekkes JR, Bos JD and Middelkoop E: Cultured fibroblasts from chronic diabetic wounds on the lower extremity (non-insulin-dependent diabetes mellitus) show disturbed proliferation. Arch Dermatol Res 291: 93-99, 1999.

12. Rowe DW, Starman BJ, Fujimoto WY and Williams RH: Abnormalities in proliferation and protein synthesis in skin fibroblast cultures from patients with diabetes mellitus. Diabetes 26: 284-290, 1977.

13. Bachschmid MM, Xu S, Maitland-Toolan KA, Ho YS, Cohen RA and Matsui R: Attenuated cardiovascular hypertrophy and oxidant generation in response to angiotensin II infusion in glutaredoxin-1 knockout mice. Free Radic Bio Med 49: $1221-1229,2010$

14. Xuan YH, Huang BB, Tian HS, Chi LS, Duan YM, Wang X, Zhu ZX, Cai WH, Zhu YT, Wei TM, et al: High-glucose inhibits human fibroblast cell migration in wound healing via repression of bFGF-regulating JNK phosphorylation. PLoS One 9: e108182, 2014.

15. Gene Ontology Consortium: The gene ontology (GO) project in 2006. Nucleic Acids Res 34: D322-D326, 2006.

16. Dupuy D, Bertin N, Hidalgo CA, Venkatesan K, Tu D, Lee D, Rosenberg J, Svrzikapa N, Blanc A, Carnec A, et al: Genome-scale analysis of in vivo spatiotemporal promoter activity in Caenorhabditis elegans. Nat Biotechnol 25: 663-668, 2007.

17. Dunnick JK, Brix A, Cunny H, Vallant M and Shockley KR: Characterization of polybrominated diphenyl ether toxicity in Wistar Han rats and use of liver microarray data for predicting disease susceptibilities. Toxicol Pathol 40: 93-106, 2012.
18. Kanehisa M and Goto S: KEGG: Kyoto Encyclopedia of Genes and Genomes. Nucleic Acids Res 28: 27-30, 2000.

19. Matthews L, Gopinath G, Gillespie M, Caudy M, Croft D, de Bono B, Garapati P, Hemish J, Hermajakob H, Jassal B, et al: Reactome knowlegdebase of human biological pathways and processes. Nucleic Acids Res 37: 619-622, 2009.

20. Draghici S, Khatri P, Tarca AL, Amin K, Done A, Voichita C, Georgescu C and Romero R: A systems biology approach for pathway level analysis. Genome Res 17: 1537-1545, 2007.

21. Livak KJ and Schmittgen TD: Analysis of relative gene expression data using real-time quantitative PCR and the 2(-Delta Delta C(T)) method. Methods 25: 402-408, 2001.

22. Lamers ML, Almeida ME, Vicente-Manzanares M, Horwitz AF and Santos MF: High glucose-mediated oxidative stress impairs cell migration. PLoS One 6: e22865, 2011.

23. Baeuerle PA: IkappaB-NF-kappaB structures: At the interface of inflammation control. Cell 95: 729-731, 1998.

24. Malinauskas T and Jones EY: Extracellular modulators of Wnt signalling. Curr Opin Struct Biol 29: 77-84, 2014.

25. Krafts KP: Tissue repair: The hidden drama. Organogenesis 6: 225-233, 2010

26. Hinz B: Masters and servants of the force: The role of matrix adhesions in myofibroblast force perception and transmission. Eur J Cell Biol 85: 175-181, 2006.

27. Greenhalgh DG: The role of apoptosis in wound healing. Int $\mathrm{J}$ of Biochem Cell Biol 30: 1019-1030, 1998.

28. Stashak TS, Farstvedt E and Othic A: Update on wound dressings: Indications and best use. Clin Tech Equine Prac 3: 148-163, 2004

29. Versteeg HH, Heemskerk JW, Levi M and Reitsma PH: New fundamentals in hemostasis. Physiological Reviews 93: 327-358, 2013.

30. Meyerson M, Gabriel S and Getz G: Advances in understanding cancer genomes through second-generation sequencing. Nat Rev Genet 11: 685-696, 2010.

31. Stulberg MJ, Lin A, Zhao H and Holley SA: Crosstalk between Fgf and Wnt signaling in the zebrafish tailbud. Dev Biol 369: 298-307, 2012.

32. Butí E, Mesquita D and Araújo SJ: Hedgehog is a positive regulator of FGF signalling during embryonic tracheal cell migration. PLoS One 9: e92682, 2014

33. Reddy BV and Irvine KD: Regulation of Hippo signaling by EGFR-MAPK signaling through Ajuba family proteins. Dev Cell 24: 459-471, 2013.

34. Flati V, Pastore LI, Griffioen AW, Satijn S, Toniato E, D'Alimonte I, Laglia E, Marchetti P, Gulino A and Martinotti S: Endothelial cell anergy is mediated by bFGF through the sustained activation of p38-MAPK and NF-kappaB inhibition. Int J Immunopathol Pharmacol 19: 761-773, 2006. 